\title{
Rifaximin use favoured micafungin-resistant Candida spp. infections in recipients of allogeneic hematopoietic cell transplantation
}

\author{
Francesca Marzuttini ${ }^{1}$ - Antonella Mancusi ${ }^{1}$ - Samanta Bonato ${ }^{1} \cdot$ Mario Griselli $^{1}$ - Sara Tricarico ${ }^{1}$. Genni Casarola ${ }^{1}$. \\ Matteo Paradiso ${ }^{1}$ - Loredana Ruggeri ${ }^{1}$ - Adelmo Terenzi ${ }^{1}$. Mara Merluzzi ${ }^{1}$. Anna Prigitano ${ }^{2}$ - Anna Maria Tortorano ${ }^{2}$. \\ Lucia Pitzurra $^{3} \cdot$ Brunangelo Falini $^{1} \cdot$ Alessandra Carotti $^{1} \cdot$ Andrea Velardi $^{1} \cdot$ Antonio Pierini $^{1}$ (I)
}

Received: 3 March 2021 / Accepted: 4 June 2021 / Published online: 28 June 2021

(c) The Author(s) 2021

\begin{abstract}
Damage to gut mucosa following conditioning regimens may favour bacterial infections that can trigger graft versus host disease (GvHD) in patients undergoing allogeneic hematopoietic stem cell transplantation (HSCT). Rifaximin, an oral and non-absorbable antibiotic, has been recently proposed as effective prophylaxis to reduce bacterial infections in the gut and consequently acute GvHD in this setting. The present study evaluated safety and outcomes of HSCT patients that were treated with rifaximin prophylaxis at Perugia University Hospital. Rifaximin prophylaxis was introduced as standard of care in HSCT patients in May 2018. We retrieved data from 118 consecutive transplants, and we compared the outcomes of rifaximin-treated patients with historical controls that did not receive antibiotic prophylaxis. While incidences of neutropenic fever, documented bacterial infections, and aGvHD were similar in the two groups, we found an increased frequency of invasive candidiasis and clinically relevant Candida spp. infections in rifaximin-treated patients (5 patients vs 1 patient, 25\% $[ \pm 0.99 \%]$ vs $1 \%[ \pm 0.01 \%], \mathrm{p}<.0001)$. Three rifaximin-treated patients experienced life-threating candidemia $(2 \mathrm{C}$. krusei, 1 C. orthopsilosis). Rifaximin was the only factor that increased the risk of Candida spp. infections. Rifaximin could have contributed to microbiome disruption which favoured an outbreak of life-threatening Candida infections. This important complication forced us to halt its use. Larger, prospective studies are needed to assess the impact of rifaximin prophylaxis on incidence of bacterial infections, aGvHD, and survival of HSCT patients.
\end{abstract}

Keywords Candida $\cdot$ Rifaximin $\cdot$ Hematopoietic stem cell transplantation $\cdot$ Microbiome $\cdot$ Prophylaxis $\cdot$ Echinocandin

\section{Introduction}

Bacterial infections are common complications during neutropenia that follows allogeneic hematopoietic stem cell transplantation (HSCT), but there is no general consensus

On November 19th, 2020, the study was registered at the local (Umbria Italian region) ethical committee (IRB) with registration number 01/2020 in CER (Comitato Etico Regionale) registry (N.: $3865 / 19)$.

Antonio Pierini

antonio.pierini@unipg.it

1 Division of Hematology and Clinical Immunology, University of Perugia, Perugia, Italy

2 Department of Biomedical Sciences for Health, Università Degli Studi Di Milano, Milan, Italy

3 Division of Microbiology, University of Perugia, Perugia, Italy regarding the need, the type, and the duration of antibiotic prophylaxis in this setting. Antibiotic prophylaxis with fluoroquinolones alone or fluoroquinolones plus metronidazole has been considered a standard of care at most institutions as it has been shown to reduce incidence of gram-negative sepses [1,2]. However, recent studies showed an increase of bacterial resistance to fluoroquinolones which questioned their efficacy [3]. Thus, some centres even abandoned prophylaxis use and opted for a pre-emptive or empiric approach to bacterial infections.

Recent studies show that intestinal microbiota regulates immune homeostasis. Loss of microbiota diversity and prevalence of enterococci have been linked to the onset of acute graft-versus-host disease (aGvHD), an immune-mediated and potentially life-threatening complication of HSCT [4, 5]. Rifaximin, an oral and almost non absorbable antibiotic which preserves intestinal microbiota diversity, can reduce enterococci and may exert anti-inflammatory activities. A 
single-centre retrospective study by Weber et al. suggested that antibiotic prophylaxis with rifaximin could reduce incidence of aGvHD and improve overall survival of transplanted patients if compared to fluoroquinolone plus metronidazole prophylaxis [6].

Our group historically chose to avoid antibiotic prophylaxis for HSCT patients because of high prevalence of fluoroquinolone-resistant bacterial infections. Following the study by Weber et al., we recently changed this policy and introduced antibiotic prophylaxis with rifaximin to reduce such infections and, possibly, incidence of aGvHD. Here we report incidence of infections and outcomes of patients that received rifaximin prophylaxis at our institution.

\section{Materials and methods}

\section{Ethics}

The study was conducted according to the revised Helsinki Declaration and was approved by Umbria Regional Hospital Institutional Review Board (IRB). Written informed consent was obtained from each patient.

\section{Demographics}

In this retrospective study, we compared outcomes of rifaximin-treated patients with historical controls at our institution. We analysed transplants performed from January 2016 to August 2019 at the University Hospital of Perugia.

We started rifaximin use in May 2018. Rifaximin $200 \mathrm{mg} \mathrm{q} 12 \mathrm{~h}$ was given from the beginning of the conditioning regimen to day +20 post-transplant. Controls received no antibiotic prophylaxis. All patients without pre-HSCT fungal disease received micafungin $50 \mathrm{mg}$ q24h as antifungal primary prophylaxis, while patients who experienced possible or probable fungal pneumonia before transplant received lyposomal amphotericin-B (L-AmB) $1-3 \mathrm{mg} / \mathrm{kg}$ q24h as secondary prophylaxis. Trimethoprimsulfamethoxazole $960 \mathrm{mg}$ three times each week was used as prophylaxis for $P$. jirovecii pneumonia starting at day +50 after transplant.

We retrieved the following information for each patient: age, gender, underlying haematologic disease, type of graft (HLA-matched related HSCT, HLA-mismatched related, unrelated), intensity of the conditioning regimen, previous Candida spp. colonization, diagnosis of fungal pneumonia before HSCT, previous exposure to echinocandins, antifungal prophylaxis, and duration of micafungin prophylaxis (Table 1). We used the EORTC (European Organization for Research and Treatment of Cancer) criteria to define invasive candidiasis [7]. Candida spp. isolates that were considered worth of systemic antifungal treatment because they were associated to specific clinical signs and/ or symptoms of disease have been defined as clinically
Table 1 Demographics of the patient population. $H L A$, human leukocyte antigen; $H S C T$, allogeneic hematopoietic stem cell transplantation; $L-A m B$, lyposomal amphotericin-B

\begin{tabular}{lllll}
\hline & Rifaximin & No prophylaxis & $p$ value & All patients \\
\hline No of patients & $21(18 \%)$ & $97(82 \%)$ & & 118 \\
Age (median) & 57 & 52 & .031 & 52.5 \\
Gender (male) & $7(33 \%)$ & $65(67 \%)$ & .004 & $72(61 \%)$ \\
Underlying hematologic disease & & & .68 & \\
Myeloid & $12(57 \%)$ & $60(62 \%)$ & & $72(61 \%)$ \\
Lymphoid & $9(43 \%)$ & $37(38 \%)$ & & $46(39 \%)$ \\
Type of graft & & & .42 & \\
HLA-matched related & $3(14 \%)$ & $20(21 \%)$ & & $23(20 \%)$ \\
HLA-mismatched related & $18(86 \%)$ & $72(74 \%)$ & & $90(76 \%)$ \\
Unrelated & 0 & $5(5 \%)$ & & $5(4 \%)$ \\
Conditioning regimen & & & .77 & $98(83 \%)$ \\
Myeloablative & $17(81 \%)$ & $81(84 \%)$ & & $20(17 \%)$ \\
Reduced-intensity or non-myeloablative & $4(19 \%)$ & $16(16 \%)$ & & $48(41 \%)$ \\
Previous Candida spp. colonization & $8(38 \%)$ & $40(41 \%)$ & .79 & $28(24 \%)$ \\
Fungal pneumonia before HSCT & $3(14 \%)$ & $25(26 \%)$ & .26 & $12(10 \%)$ \\
Previous exposure to echinocandins & $3(14 \%)$ & $9(9 \%)$ & .49 & .009 \\
Antifungal prophylaxis & & & & $93(79 \%)$ \\
Primary (with micafungin) & $21(100 \%)$ & $72(74 \%)$ & & $25(21 \%)$ \\
Secondary (with L-AmB) & 0 & $25(26 \%)$ & & $28(3-102)$ \\
Duration of micafungin prophylaxis & $28(18-102)$ & $28(3-72)$ & & \\
$\quad$ (median days, range) & & & & \\
\hline
\end{tabular}


relevant Candida spp. infections and were included in the analysis.

\section{Statistical analysis}

Demographics and prognostic variables were compared using the $\chi^{2}$ test for categorical variables, and the Student $t$ test or Mann-Whitney test for continuous variables. The Kaplan-Meier method evaluated overall and disease-free survival. A log-rank test assessed rifaximin impact on survival. Cumulative incidence (CI) estimates were used for relapse and non-relapse mortality (NRM) because they were considered competing risks. CI of aGvHD or Candida infections was calculated using death from any cause as competing risk. Gray test compared impact of rifaximin on univariate competing risk outcomes. Multivariate analyses assessed the impact of rifaximin on incidence of clinically relevant Candida spp. infections. Different demographic features (Table 1) were included as variables using a Cox regression model in a conditional forward stepwise fashion to identify factors with a significant impact on outcomes. All $\mathrm{p}$ values were 2 -sided and considered significant at $\mathrm{p}<0.05$.

\section{Results}

We retrieved data from 118 consecutive transplants. Twentyone received rifaximin, while 97 received no antibiotic prophylaxis. Engraftment occurred in all, but in six patients ( 1 received rifaximin, 5 no antibiotic prophylaxis, $p=$ not significant, NS). Patients were well-matched for underlying hematologic disease, type of graft, intensity of the conditioning regimen, previous Candida spp. colonization, diagnosis of fungal pneumonia before HSCT, previous exposure to echinocandins, and duration of micafungin prophylaxis. Patients who received rifaximin were slightly older than patients in the control group, and female/male ratio was slightly higher. None of the 21 patients who received rifaximin was treated with L-AmB as antifungal prophylaxis, while L-AmB was used as secondary antifungal prophylaxis in 25 control transplants. Patient characteristics are detailed in Table 1.

Incidences of neutropenic fever (100\% in rifaximintreated patients vs $96 \%$ in control patients, $p=N S$ ), documented sepsis $(25 \%$ vs $33 \%, \mathrm{p}=\mathrm{NS})$, and use of carbapenems $(80 \%$ vs $75 \%, \mathrm{p}=\mathrm{NS})$ were similar between rifaximin-treated patients and historical controls (Table 2). Also, aGvHD occurred with a similar incidence between the two groups (CI in rifaximin-treated patients: $35 \%$ [ $\pm 1.22 \%$; $\mathrm{CI}$ in control patients: $35 \%$ [ $\pm 0.25 \%], \mathrm{p}=\mathrm{NS}$ ) (supplementary Fig. 1). Surprisingly, we found clinically relevant $\mathrm{Can}$ dida spp. infections (5 patients vs 1, 25\% [ $\pm 0.99 \%]$ vs $1 \%$
Table 2 Outcomes of transplanted patients according to the use of rifaximin prophylaxis. Percentage of events is calculated on the total of engrafted patients. HSCT, allogeneic hematopoietic stem cell transplantation; $C M V$, Cytomegalovirus; $E B V$, Epstein-Barr virus; $G v H D$, graft versus host disease

\begin{tabular}{|c|c|c|c|c|}
\hline & Rifaximin & No prophylaxis & $p$ value & All patients \\
\hline Engraftment & $20 / 21(95 \%)$ & $92 / 97(95 \%)$ & .94 & $112 / 118(95 \%)$ \\
\hline Neutropenic fever & $20(100 \%)$ & $88(96 \%)$ & .34 & $108(96 \%)$ \\
\hline Documented sepses & $5(25 \%)$ & $30(33 \%)$ & .51 & $35(31 \%)$ \\
\hline \multicolumn{5}{|l|}{ Bacterial isolate } \\
\hline Escherichia coli & $2(40 \%)$ & $16(53 \%)$ & .41 & $18(51 \%)$ \\
\hline Enterococcus fecium and faecalis & 0 & $2(6 \%)$ & .51 & $2(6 \%)$ \\
\hline Other gram positive & $2(40 \%)$ & $10(33 \%)$ & .91 & $12(34 \%)$ \\
\hline Other gram negative & $1(20 \%)$ & $2(6 \%)$ & .48 & $3(9 \%)$ \\
\hline Use of carbapenems & $16(80 \%)$ & $69(75 \%)$ & .64 & $85(76 \%)$ \\
\hline \multicolumn{5}{|l|}{ Fungal infections post HSCT } \\
\hline Clinically relevant candidiasis & $5(25 \%)$ & $1(1 \%)$ & $<.0001$ & $6(5 \%)$ \\
\hline Invasive candidiasis & $3(15 \%)$ & $0(0 \%)$ & .0002 & $3(3 \%)$ \\
\hline Other & $6(30 \%)$ & $40(43 \%)$ & .27 & $46(41 \%)$ \\
\hline \multicolumn{5}{|l|}{ Viral reactivations } \\
\hline $\mathrm{CMV}$ & $11(55 \%)$ & $54(59 \%)$ & .76 & $65(58 \%)$ \\
\hline EBV & $5(25 \%)$ & $33(36 \%)$ & .35 & $38(34 \%)$ \\
\hline \multicolumn{5}{|l|}{ Acute GvHD } \\
\hline Grade II-IV & $7(35 \%)$ & $32(35 \%)$ & .98 & $39(35 \%)$ \\
\hline Grade III-IV & $4(20 \%)$ & $21(23 \%)$ & .78 & $25(22 \%)$ \\
\hline Steroid refractory & $3(15 \%)$ & $19(21 \%)$ & .42 & $22(20 \%)$ \\
\hline
\end{tabular}


[ $\pm 0.01 \%], \mathrm{p}<0.0001)$ (Fig. 1) and invasive candidiases (3 vs $0,15 \%[ \pm 0.67 \%]$ vs $0 \%$ [ $\pm 0 \%], p=0.0002$ ) (supplementary Fig. 2) were more frequent in rifaximin-treated patients. Incidences of other fungal infections and viral (Cytomegalovirus, CMV and Epstein-Barr virus, EBV) reactivations did not differ in rifaximin-treated patients and controls (other fungal infections: $30 \%$ vs $43 \%$, p =NS; CMV: $55 \%$ vs $59 \%$, $\mathrm{p}=\mathrm{NS}$; EBV: $25 \%$ vs $36 \%, \mathrm{p}=\mathrm{NS})$. Three of the 5 rifaximin-treated patients with Candida spp. infection experienced life-threatening invasive candidiasis ( 3 candidaemias, 2 of which by $C$. krusei, 1 by $C$. orthopsilosis). Details about Candida spp. isolates are reported in supplementary table 1. All Candida infections happened early after transplant (medium time 26 days, range 5-91). Multivariate analysis confirmed rifaximin was the only factor that increased the risk of Candida spp. infection as such risk was not dependent upon age, gender, underlying hematologic disease, type of graft, intensity of the conditioning regimen, and previous Candida spp. colonization (supplementary table 2). To assess whether antifungal prophylaxis had an impact on Candida spp. infections, patients that received secondary prophylaxis with L-Amb were excluded from the analysis as none of them was treated with rifaximin. Rifaximin prophylaxis was confirmed as the only risk factor for the development of invasive candidiasis and clinically relevant Candida spp. infection (supplementary Fig. 3).

L-AmB was chosen for the treatment of micafunginresistant Candida infections in 5 of the 6 patients, while voriconazole was used to treat one of them. All the patients resolved the Candida spp. infections. Candida infections did not impact on disease-free survival (55\% in rifaximintreated patients vs $57 \%$ in patients that received no antibiotic prophylaxis, $\mathrm{p}=\mathrm{NS}$ ) (supplementary Fig. 4) and overall survival $(60 \%$ vs $61 \%, p=N S)$ (supplementary Fig. 5) probably because of the low number of rifaximin-treated patients. In fact, the increase of clinically relevant Candida infections forced us to halt rifaximin use.

\section{Discussion}

The present study shows rifaximin prophylaxis could favour Candida spp. infections in patients who undergo allogeneic HSCT and are treated with echinocandin-based antifungal prophylaxis. The low number of patients that were treated with rifaximin prophylaxis at our institution
Fig. 1 Cumulative incidence of clinically relevant Candida spp. infections. Incidence of clinically relevant Candida spp. infections was higher in rifaximin-treated patients $(25 \%$ $[ \pm 0.99 \%]$ vs $1 \%[ \pm 0.01 \%])$

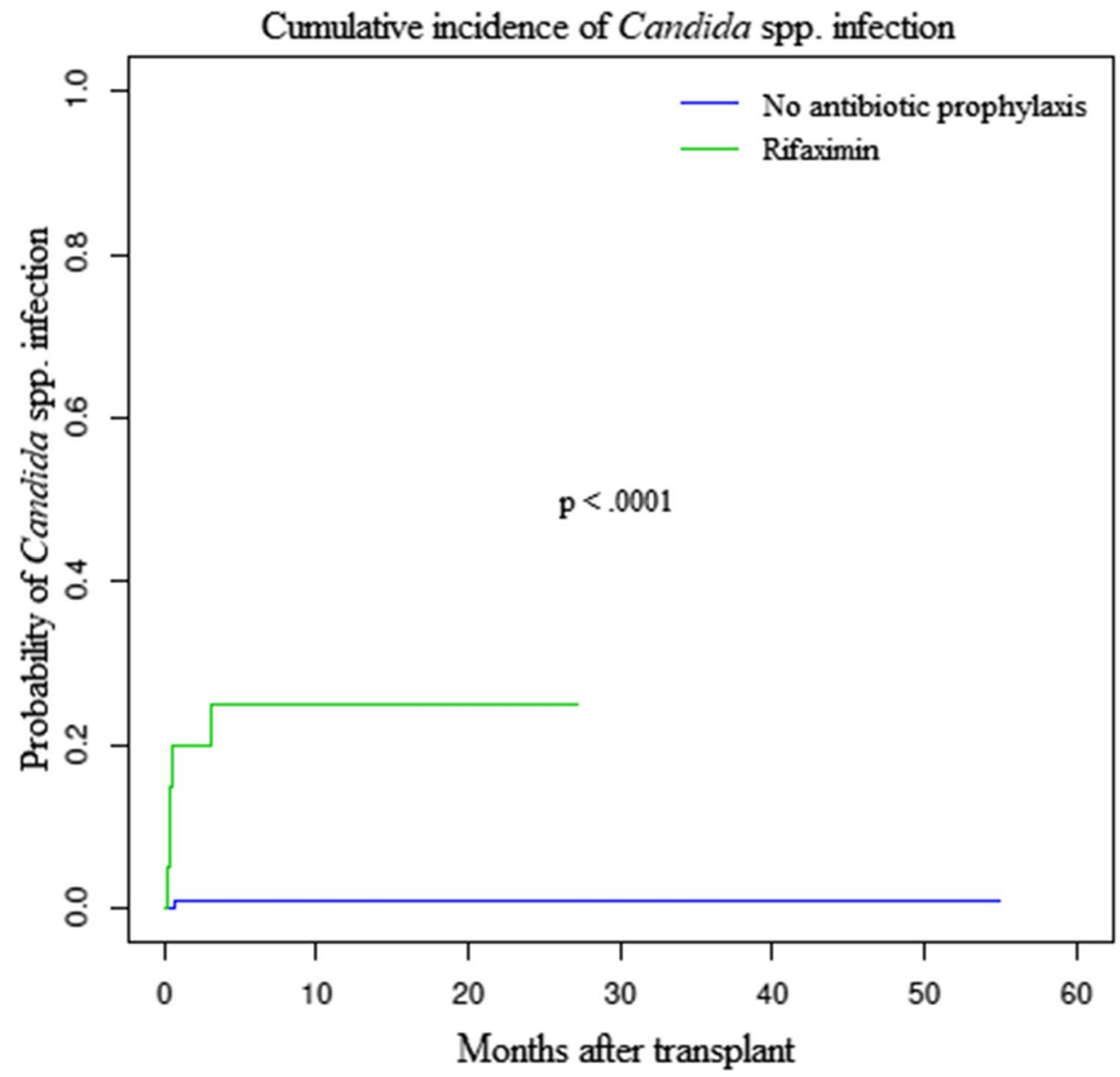


does not allow us to evaluate its efficacy in preventing bacterial infections and, consequently, its possible impact on incidence of aGvHD. Nevertheless, the rise of Candida spp. infections in this small subset of patients and the clinical impact of such complications urged us to quickly modify our anti-infective prophylactic strategies in allogeneic HSCT recipients. The present report underlines the clinical need to adjust anti-infective prophylaxes to local epidemiology taking into account drug interactions and concomitant effects on microbiome. Moreover, the present study demonstrates the clinical relevance of fungal dysbiosis in the context of HSCT and urges the scientific community to explore the impact of fungal microbiota on HSCT outcomes. In this retrospective study, we could not investigate whether rifaximin impacted on microbiome diversity. However, despite that the rate of bacterial (e.g. E. coli and enterococci) infections did not differ between rifaximin-treated patients and controls, it is likely that rifaximin played a relevant role in modifying environmental factors that favoured this Candida outbreak in echinocandin-treated patients. Indeed, we did not observe further Candida spp. infections after rifaximin use was suspended in patients that received the same micafungin prophylaxis regimen.

In fact, all the 6 Candida spp. infections occurred in patients that were receiving prophylactic micafungin. Development of echinocandin resistance in Candida spp. infection is a rare phenomenon. It is probably favoured by prolonged echinocandin exposure and biofilm formation which determines poor drug penetration and consequently strong selective pressure $[8,9]$. The mechanism of acquired resistance to echinocandin therapy involves genetic mutations in specific hot spot regions of $f k s$ target genes, and it is usually associated with an increase in echinocandin minimum inhibitory concentration (MIC) in vitro [8]. Echinocandin MIC elevation, response to echinocandin in vivo, and clinical outcomes may be different according to the type of $f k s 1$ mutation [10]. Thus, we analysed the genotype of the two C. krusei isolates and we found that both presented the L701M mutation in the $f k s 1$ gene. While other $f k s 1$ mutations are clearly associated to echinocandin resistance, the role of L701M mutation is still unclear. This mutation has been previously identified in association with elevated caspofungin MIC if coupled to other mutations with an ascertained role in echinocandin resistance $[11,12]$. On the other hand, L701M mutation alone may not have an impact on MIC values [13]. In fact, all our isolates were sensitive to echinocandins at the antifungal susceptibility testing while they showed resistance to prophylactic doses of micafungin in vivo. Thus, it is possible that L701M mutation may favour in vivo resistance to relatively low dose echinocandin regimens, at least in Candida krusei infections. Weber et al. did not specify the antifungal prophylaxis used and did not report the rate of Candida infections in their study [6]. Indeed, safety of rifaximin in HSCT recipients needs to be explored when coupled with antifungal prophylaxis other than micafungin.

In conclusion, despite that our study retrieved data from a relatively low number of patients with a possible bias due to local epidemiology and to a specific strategy in antimicrobial prophylaxis and treatment, it shows prophylactic use of rifaximin in HSCT patients may result in an increase of Candida spp. infections. Further and wider studies are required to investigate the role of rifaximin in the modulation of microbiota diversity, the development of bacterial and Candida infections, and its final effect on patients undergoing HSCT.

Supplementary Information The online version contains supplementary material available at https://doi.org/10.1007/s00277-021-04569-x.

Acknowledgements We thank Comitato per la Vita Daniele Chianelli for its constant support to transplanted patients, Associazione Italiana per la Ricerca sul Cancro for the support to research in hematopoietic stem cell transplantation, and all the residents and nurses.

Author contribution FM provided clinical care and wrote the manuscript; AM and MM analysed data; SB, MG, ST, GC, MP, LR, AT, and AC provided clinical care and reviewed the manuscript; APr, AMT, and LP performed microbiological analysis and reviewed the manuscript; BF provided overall guidance; AV provided clinical care and overall guidance; APi provided clinical care and guidance, and wrote the manuscript.

Funding Open access funding provided by Università degli Studi di Perugia within the CRUI-CARE Agreement. AP is supported by Associazione Italiana per la Ricerca sul Cancro (AIRC), Start-Up grant N.20456.

Data availability The authors will provide data after verified and detailed institutional request by professional members. Data will be available starting 3 months within 1 year from the date of publication. Request needs to be made to antonio.pierini@unipg.it.

Code availability Not applicable.

\section{Declarations}

Ethics approval The study was conducted according to the revised Helsinki Declaration and was approved by Umbria Regional Hospital Institutional Review Board (IRB).

Consent to participate Written informed consent was obtained from each patient.

Consent for publication Written informed consent was obtained from each patient.

Conflict of interest The authors declare no competing interests.

Open Access This article is licensed under a Creative Commons Attribution 4.0 International License, which permits use, sharing, adaptation, distribution and reproduction in any medium or format, as long as you give appropriate credit to the original author(s) and the source, 
provide a link to the Creative Commons licence, and indicate if changes were made. The images or other third party material in this article are included in the article's Creative Commons licence, unless indicated otherwise in a credit line to the material. If material is not included in the article's Creative Commons licence and your intended use is not permitted by statutory regulation or exceeds the permitted use, you will need to obtain permission directly from the copyright holder. To view a copy of this licence, visit http://creativecommons.org/licenses/by/4.0/.

\section{References}

1. Horton LE, Haste NM, Taplitz RA (2018) Rethinking antimicrobial prophylaxis in the transplant patient in the world of emerging resistant organisms - where are we today ? Curr Hematol Malig Rep 13(1):59-67

2. Peled JU, Jenq RR, Holler E, van den Brink MRM (2016) Role of gut flora after bone marrow transplantation. Nat Microbiol 1:16036

3. Averbuch D, Tridello G, Hoek J et al (2017) Antimicrobial resistance in gram-negative rods causing bacteremia in hematopoietic stem cell transplant recipients: intercontinental prospective study of the Infectious Disease Working Party of the European Bone Marrow Transplantation group. Clin Infect Dis 65(11):1819-1828

4. Peled JU, Gomes ALC, Devlin SM et al (2020) Microbiota as predictor of mortality in allogeneic hematopoietic-cell transplantation. N Engl J Med 382(9):822-834

5. Staffas A, Burgos da Silva M, van den Brink MRM (2017) The intestinal microbiota in allogeneic hematopoietic cell transplant and graft versus-host disease. Blood 129(8):927-933

6. Weber D, Oefner PJ, Dettmer K et al (2016) Rifaximin preserves intestinal microbiota balance in patients undergoing allogeneic stem cell transplantation. Bone Marrow Transplant 51(8):1087-1092
7. Donnelly JP, Chen SC, Kauffman CA et al (2020) Revision and update of the consensus definitions of invasive fungal disease from the European Organization for Research and Treatment of Cancer and the Mycoses Study Group Education and Research Consortium. Clin Infect Dis 71(6):1367-1376

8. Forastiero A, Garcia-Gil V, Rivero-Menendez O et al (2015) Rapid development of Candida krusei echinocandin resistance during caspofungin therapy. Antimicrob Agents Chemother 59(11):6975-6982

9. Perlin DS (2015) Echinocandin resistance in Candida. Clin Infect Dis 61(S6):S612-617

10. Lackner M, Tscherner M, Schaller M et al (2014) Positions and numbers of $F K S$ mutations in Candida albicans selectively influence in vitro and in vivo susceptibilities to echinocandin treatment. Antimicrob Agents Chemoter 58(7):3626-3635

11. Desnos-Ollivier M, Bretagne S, Raoux D, Hoinard D, Dromer F, Dannaoui E (2008) Mutations in the fks1 gene in Candida albicans, C.tropicalis, and $\mathrm{C}$ krusei correlate with elevated caspofungin MICs uncovered in AM3 medium using the method of the European Committee on Antibiotic Susceptibility Testing. Antimicrob Agents Chemother 52(9):3092-8

12. Prigitano A, Esposto MC, Cogliati M, Pitzurra L, Santamaria C, Tortorano AM (2014) Acquired echinocandin resistance in a Candida krusei blood isolate confirmed by mutations in $f k s 1$ gene. New Microbiol 37(2):237-240

13. Lallitto F, Prigitano A, Mangione F et al (2018) Presence of L701M mutation in the FKS1 gene of echinocandin-susceptible Candida krusei isolates. Diagn Microbiol Infect Dis 92(4):311-314

Publisher's note Springer Nature remains neutral with regard to jurisdictional claims in published maps and institutional affiliations. 